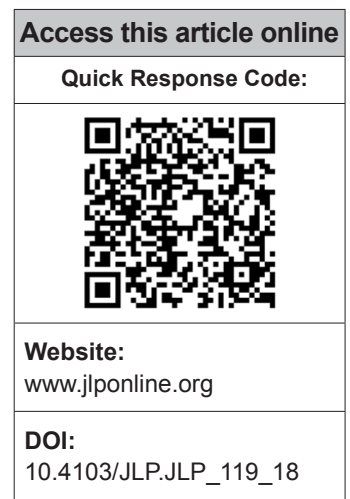

Department of Pathology, Postgraduate Institute of Medical Education and Research,

Dr. Ram Manohar Lohia Hospital, New Delhi, India

Address for correspondence: Dr. Purnima Malhotra, Department of Pathology, Room No. 323

$3^{\text {rd }}$ Floor, OPD Building, Dr. RML Hospital, Baba

Kharak Singh Maarg, New Delhi - 110 001, India. E-mail: purnimapaliwal@ gmail.com

Submission: 19-09-2018 Accepted: 22-11-2018

\section{Role of immunohistochemistry in diagnosis of a rare bladder tumor}

\author{
Manjari Kishore, Purnima Malhotra, Minakshi Bhardwaj
}

\title{
Abstract:
}

Neuroendocrine tumors (NETs) are commonly located in the respiratory and gastrointestinal tract; however, these tumors can rarely be found in the urinary bladder. NETs comprise $<1 \%$ of all bladder tumors; usually intermixed with urothelial carcinoma and its variants. We report a case of primary neuroendocrine carcinoma of urinary bladder in an adult female with a history of smoking and hematuria. The present case highlights the importance of immunohistochemistry in arriving at an accurate diagnosis and thereby differentiating this tumor from other clinical mimics.

Key words:

Contrast-enhanced computed tomography, hematuria, immunohistochemistry, neuroendocrine malignancy, urinary bladder

\section{Introduction}

U rothelial carcinoma is the most common malignancy found in urinary bladder, but rare neoplasms like neuroendocrine tumors (NETs) do occur at this location. ${ }^{[1,2]}$ NETs are epithelial tumors with neuroendocrine differentiation and can be found in most organs in the human body including the urinary bladder. In bladder, NETs are usually found intermixed with urothelial carcinoma and its variants. Primary neuroendocrine carcinomas of the urinary bladder account for $<1 \%$ of all bladder tumors..$^{[1-3]}$ It was first reported by Cramer in 1981. ${ }^{[2]}$ The most common presentation is hematuria. The cell of origin is thought to be multipotent stem cell. These are aggressive tumors with poor median survival age; hence, an early diagnosis is crucial. Here, we present a rare case of neuroendocrine carcinoma in a 50-year-old female, with diagnostic histopathological and immunohistochemistry (IHC) findings.

This is an open access journal, and articles are distributed under the terms of the Creative Commons Attribution-NonCommercial-ShareAlike 4.0 License, which allows others to remix, tweak, and build upon the work non-commercially, as long as appropriate credit is given and the new creations are licensed under the identical terms.

For reprints contact: reprints@medknow.com

\section{Case Report}

A 50-year-old female patient presented to hospital with chief complaints of hematuria for the past 2 months. There was no history of associated urinary urgency, increased frequency, or dysuria. No history of any chronic illness was found in the patient. However, history of smoking was present.

Complete blood count and biochemical parameters, including the liver and kidney function test, serum electrolytes, and blood sugar levels of the patient, were within the normal limits. Routine urine examination revealed numerous pus cells and red blood cells. Ultrasonography (USG) of whole abdomen showed evidence of well-defined focal lesion measuring $3.7 \mathrm{~cm} \times 3.4 \mathrm{~cm} \times 2.6 \mathrm{~cm}$ arising from the wall of the urinary bladder and projecting into the lumen, predominantly on the left side with obstruction of the left ureter with left hydroureter and hydronephrosis. Contrast-enhanced computed tomography confirmed the USG findings by revealing well defined heterogeneously enhancing lobulated mass along the left lateral wall of the bladder [Figure 1].

How to cite this article: Kishore M, Malhotra $\mathrm{P}$, Bhardwaj M. Role of immunohistochemistry in diagnosis of a rare bladder tumor. J Lab Physicians 2019;11:91-3. 


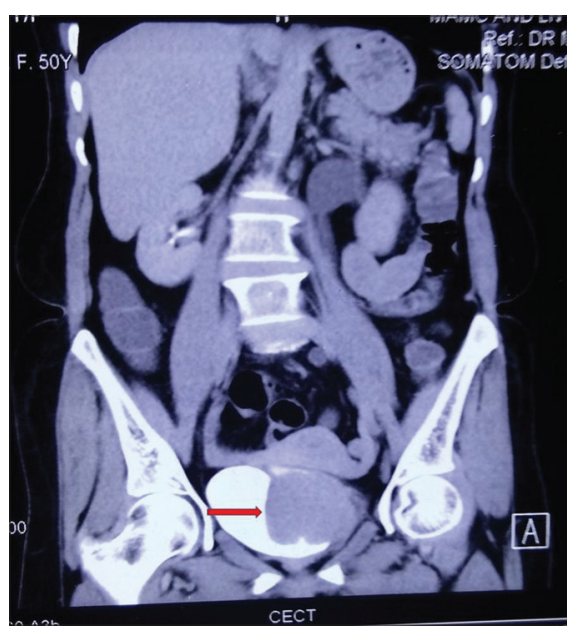

Figure 1: Contrast-enhanced computed tomography showing a well-defined heterogeneously enhancing lobulated mass along the left lateral wall of the bladder (marked with red arrow)

Transurethral resection of bladder tumor was done and biopsy was sent from the tumor as well as from the deep muscle. Section showed a highly infiltrative tumor, with extensive infiltration into the deep muscle and focal necrosis. Tumor cells were arranged in sheets and showed scant cytoplasm, pleomorphic, angulated and hyperchromatic nuclei, fine granular chromatin, and inconspicuous nucleoli [Figure 2a and b]. Lymphovascular invasion was also noted. No dysplasia was noted in the overlying epithelium. IHC showed strong positivity of tumor cells for CD56 [Figure 2c] and focal positivity for synaptophysin [Figure 2d]. Tumor cells were negative for CK7, CK20, and uroplakin.

Based on the clinical, radiological, histological, and immunohistochemical findings, a diagnosis of Neuroendocrine carcinoma/small cell carcinoma of urinary bladder was made. The patient was advised radical cystectomy; however, she was lost to follow-up.

\section{Discussion}

Among urinary bladder malignancies, NETs comprise a very small percentage. Primary neuroendocrine carcinoma of the bladder is an extremely rare bladder malignancy with a mean frequency of approximately $0.7 \%-1 \%{ }^{[2-5]}$ Most patients are male, with male:female ratio of 5:1. The age of the presentation ranges from 32 to 91 years with the mean age of 67 years. NETs range from low-grade carcinoid tumors to high-grade small and large cell neuroendocrine carcinomas. Small cell neuroendocrine carcinomas (SCNC) comprise $<\%$ of all the primary bladder malignancies. These tumors are mostly reported in elderly population with male predominance. Usually, the neuroendocrine pattern has been found in coexistence with transitional, squamous, or undifferentiated tumor cells..$^{[5-7]}$ Most of these cases

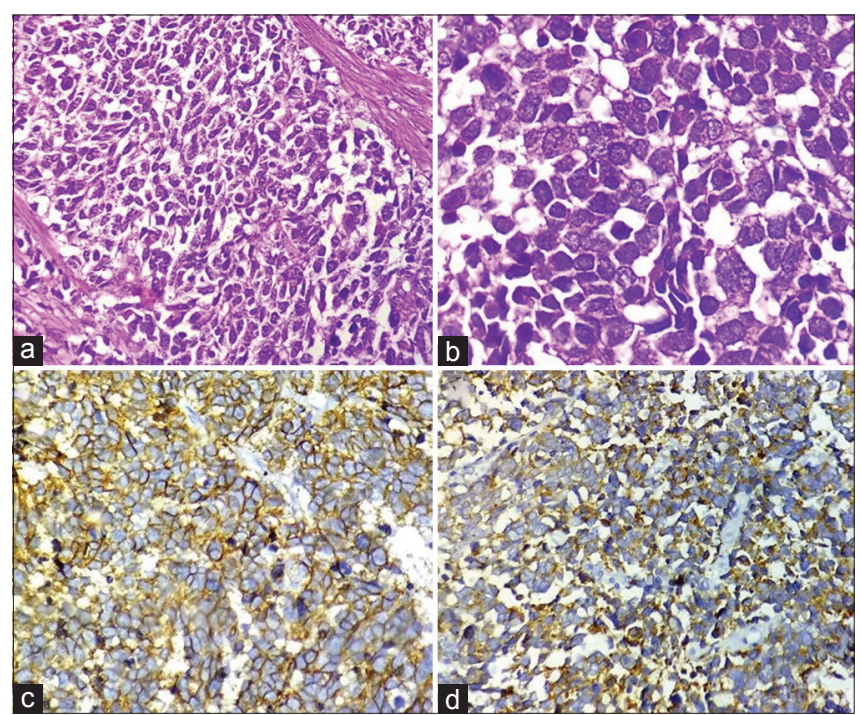

Figure 2: (a) Section showing a highly cellular tumor arranged in sheets and composed of small cells with high nuclear-cytoplasmic ratio ( $H$ and $E, \times 400)$. (b) High power view of tumor cells revealing scant cytoplasm, pleomorphic, angulated and hyperchromatic nuclei, finely granular chromatin, and inconspicuous nucleoli ( $\mathrm{H}$ and $\mathrm{E}, \times 1000)$. (c and d) Tumor cells showing positivity for CD56 (c, ×400) and synaptophysin ( $d, \times 400)$

were previously diagnosed as high grade transitional cell carcinoma (TCC), undifferentiated carcinoma, or malignant lymphoma. Pure forms of SCNC are rare and less likely associated with paraneoplastic syndromes. Patients usually present in stage III and IV with the complaint of painless hematuria.

Various theories have been put forth regarding its origin..$^{[2-5]}$ The common consensus is that it can arise from urothelial cancer stem cells as well as neuroendocrine cells located in the basal urothelial layer. These cells are also known as enterochromaffin cells and are widely distributed throughout the body. They have been described in the urinary bladder (especially in the trigone), the urethra, and the renal pelvis.

The presentation of the patients with NETs is like those of other bladder malignancies like TCC with hematuria being the most common symptom followed by dysuria. ${ }^{[4-8]}$ Other signs and symptoms can be urinary obstruction, abdominal pain, urinary tract infection, and weight loss along with rare cases of paraneoplastic syndromes. Our patient presented with gross hematuria.

Grossly, tumors are polypoidal with size varying from 4 to $10 \mathrm{~cm}$ and are usually located in the lateral wall and fundus of the bladder and rarely in the trigone region. In our case, tumor was polypoidal, measuring $3.7 \mathrm{~cm} \times 3.4 \mathrm{~cm} \times 2.6 \mathrm{~cm}$ and located in the left wall of the bladder with projection into the lumen and causing obstruction at the left ureteropelvic junction, thereby causing left hydroureter and left-sided hydronephrosis. 
Histology reveals a cellular tumor composed of nests of small round to angulated malignant cells with scant cytoplasm, pyknotic round-to-oval nuclei with prominent nuclear molding and evenly dispersed fine granular chromatin. Mitosis is usually present and can be brisk. Sometimes, tumor rosettes can also be seen along with tumor necrosis. In our case, the tumor showed similar histology. Few studies have revealed admixture of NETs with TCC, adenocarcinoma and even squamous cell carcinoma. ${ }^{[6-8]}$ However, in our case, there was no association with other tumor types on biopsy.

While evaluating these tumors, various differential diagnosis which should be kept in mind are inverted papilloma, nested variant of urothelial carcinoma, adenocarcinoma, paraganglioma, and a metastatic tumor. Although at times, histology may not help in differentiating these tumors, IHC plays an important role in such cases. Metastasis from a primary carcinoid of the appendix should be ruled out as it is more common than bladder carcinoid. NETs will show positivity for CD56, Neuron-specific enolase, synaptophysin, chromogranin, serotonin, CK, S-100 protein, thyroid transcription factor-1, epidermal growth factor receptor, and C-KIT. In the present case, tumor cells were immunoreactive for CD56 and synaptophysin, favoring NET. CK7, CK20, and uroplakin were negative ruling out possible co-existing TCC or any other epithelial malignancy in the biopsy sample.

These are aggressive tumors with the poor prognostic outcome; median survival in such patients being approximately 24 months. Combined therapy is required comprising radical resection along with radiotherapy and chemotherapy. Chemotherapy combination of etoposide and platinum-based drugs is the treatment of choice both in the adjuvant and neoadjuvant setting. ${ }^{[7-9]}$ Role of neoadjuvant chemotherapy is well established in surgically operable cases and has an increased 5 years survival as compared to patients in which only surgery has been done. Our patient was lost to follow-up and hence, no further comments can be given on prognosis of this tumor.

\section{Conclusion}

Neuroendocrine carcinomas are rare primary bladder tumors which usually present in an advanced stage.
Accurate and prompt recognition of this rare tumor is increasingly important because of its obvious prognostic and therapeutic differences from the more common urothelial malignancies. Role of histology in conjunction with IHC is very important as highlighted in the present case report.

\section{Declaration of patient consent}

The authors certify that they have obtained all appropriate patient consent forms. In the form the patient(s) has/have given his/her/their consent for his/her/their images and other clinical information to be reported in the journal. The patients understand that their names and initials will not be published and due efforts will be made to conceal their identity, but anonymity cannot be guaranteed.

\section{Financial support and sponsorship}

Nil.

\section{Conflicts of interest}

There are no conflicts of interest.

\section{References}

1. Chilari GA, Prabhala S, Gutta S, Erukkambattu J. Primary small cell neuroendocrine carcinoma of the urinary bladder. J NTR Univ Health Sci 2015;4:136-8.

2. Cramer SF, Aikawa M, Cebelin M. Neurosecretory granules in small cell invasive carcinoma of the urinary bladder. Cancer 1981;47:724-30.

3. Choong NW, Quevedo JF, Kaur JS. Small cell carcinoma of the urinary bladder. The mayo clinic experience. Cancer 2005;103:1172-8.

4. Cheng L, Pan CX, Yang XJ, Lopez-Beltran A, MacLennan GT, Lin $\mathrm{H}$, et al. Small cell carcinoma of the urinary bladder: A clinicopathologic analysis of 64 patients. Cancer 2004;101:957-62.

5. Bex A, de Vries R, Pos F, Kerst M, Horenblas S. Long-term survival after sequential chemoradiation for limited disease small cell carcinoma of the bladder. World J Urol 2009;27:101-6.

6. Abrahams NA, Moran C, Reyes AO, Siefker-Radtke A, Ayala AG. Small cell carcinoma of the bladder: A contemporary clinicopathological study of 51 cases. Histopathology 2005;46:57-63.

7. Kouba E, Cheng L. Neuroendocrine tumors of the urinary bladder according to the 2016 World Health Organization classification: Molecular and clinical characteristics. Endocr Pathol 2016;27:188-99.

8. Ulamec M, Krušlin B. Neuroendocrine tumors in the urinary bladder. Endocr Oncol Metab 2016;14:42-9.

9. Sehgal SS, Wein AJ, Bing Z, Malkowicz SB, Guzzo TJ. Neuroendocrine tumor of the bladder. Rev Urol 2010;12:e197-201. 